\title{
Global Exponential Stability of FAST TCP
}

\author{
Joon-Young Choi, Kyungmo Koo, David X. Wei, Jin S. Lee, and Steven H. Low
}

\begin{abstract}
We consider a single-link multi-source network with the FAST TCP sources. We propose a continuous-time dynamic model for the FAST TCP sources and a static model to describe the queuing delay behavior at the link. The proposed model turns out to be in a form revealing the network feedback delay, which allows us to analyze FAST TCP in due consideration of the network feedback delay. Based on the proposed model, we show the boundedness of both each source's congestion window and the queuing delay at the link; and the global exponential stability under a trivial condition that each source's congestion control parameter $\alpha$ is positive. The simulation results illustrate the validity of the proposed model and the global exponential stability of FAST TCP.
\end{abstract}

\section{INTRODUCTION}

Internet congestion control is a distributed feedback control algorithm to allocate network capacity among competing users according to a specified strategy. Internet congestion control has been commonly implemented by TCP Reno and its variants, which control their congestion window based on the well-known additive increase multiplicative decrease (AIMD) mechanism [1] [2]. It is, however, shown that these algorithms are not scalable as the delay-bandwidth product of the network becomes larger [3] [4] [5] because additive increasing is too slow and multiplicative decreasing is too severe in the large delay-bandwidth product network.

To cope with this problem, several congestion control algorithms have been proposed for high speed networks: HSTCP [6], STCP [7], FAST TCP [5], and BIC TCP [8]. Among them, FAST TCP has a feature that the queuing delay is used as a congestion measure. While the packet loss that is used as a congestion measure in TCP Reno has only binary information about the congestion, the queuing delay indicates a level of congestion, which means how far the current state is from the equilibrium. Accordingly, the congestion control mechanism operated by using the queuing delay as a congestion measure is more responsive to the network congestion and makes the network state be always around the equilibrium as long as the algorithm is stable [5].

Even though extensive experiments of FAST TCP have been conducted and the results are promising [5], the stability property of FAST TCP has not been sufficiently studied yet. It is shown in [5] that FAST TCP in a single-link network is locally asymptotically stable neglecting the network feedback delay. A sufficient condition for the local

J.-Y. Choi is with the Department of Electronic Engineering, Pusan National University, Korea; Email: jyc@pusan.ac.kr. K. Koo and J. S. Lee are with the Department of Electronic and Electrical Engineering, Pohang University of Science and Technology, Korea; Email: \{pumpkins,jsoo\}@postech.ac.kr. D. X. Wei and S. H. Low are with the Departments of Computer Science and Electrical Engineering, California Institute of Technology; Email: \{weixl,slow\}@ caltech.edu. asymptotic stability of FAST TCP in the general multi-link multi-source network was achieved considering the network feedback delay in [9]. The global stability property of FAST TCP was analyzed in [10], and a sufficient condition for the global asymptotic stability of FAST TCP in a single-link single-source network was established in the presence of the network feedback delay.

In this paper, we examine the global stability property of a single-link multi-source network with the FAST TCP sources in the presence of the network feedback delay. We propose a continuous-time dynamic model for the FAST TCP sources and a static model for the queuing delay dynamics. The proposed model turns out to adequately describe the network feedback delay between each source's congestion window and the queuing delay measured at the source. Based on the model, we establish that FAST TCP is globally exponentially stable even in the presence of the network feedback delay as long as each source's congestion control parameter $\alpha$ is positive.

This paper is organized as follows. Section II presents a dynamic model for the single-link multi-source network with the FAST TCP sources. Section III analyzes the global boundedness property of FAST TCP. Section IV shows the global exponential stability of the FAST TCP network. Section V provides the simulation results. Section VI makes conclusions.

\section{NeTwOrK MODEL}

In this section, we develop a network model to describe the behavior of FAST TCP. Usually, the models for TCP have been based on the fluid flow model, which is suitable for the rate-based TCP algorithms because the sending rate of the source is chosen as the state variable for the source in the model [4]. FAST TCP, however, is a window-based algorithm, and we construct the network model by choosing the state variable at the source as the congestion window and using the basic structure of the fluid flow model.

We consider a single-link multi-source network, where a single communication link is shared by $N$ FAST TCP sources. The link has a finite transmission capacity $c$ and is assumed to have infinite buffering storage. Each source is indexed by $i(1 \leq i \leq N)$. Associated with the link is the queuing delay $p(t)$ and with the source $i$ is the congestion window $w_{i}(t)$. We assume that the source $i$ observes the queuing delay $q_{i}(t)$ as a feedback signal in its path:

$$
q_{i}(t):=p\left(t-\tau_{i}^{b}\right),
$$

where $\tau_{i}^{b}$ denotes the backward delay in the feedback path from link to source $i$, and the link observes the aggregated 
congestion window

$$
y(t):=\sum_{i} w_{i}\left(t-\tau_{i}^{f}\right),
$$

where $\tau_{i}^{f}$ denotes the forward delay from source $i$ to link. The round trip time (RTT) $T_{i}(t)$ is defined for each source $i$ as $T_{i}(t):=d_{i}+q_{i}(t)$, where $d_{i}$ is the constant round trip propagation time. The RTT is assumed to be $T_{i}(t)=$ $\tau_{i}^{f}(t)+\tau_{i}^{b}$, where $\tau_{i}^{f}(t)$ is time-varying and $\tau_{i}^{b}$ is a constant. This assumption is reasonable if we consider that the timevarying part $q_{i}(t)$ of $T_{i}(t)$ can be totally included in the forward delay $\tau_{i}^{f}(t)$.

Taking a close look at the FAST TCP implementation of $n s-2$ simulator [11], we can obtain the pseudo-code for periodically updating the congestion window:

$$
\mathrm{w}(t+1)=\frac{1}{2}\left(\mathrm{w}(t)+\frac{\mathrm{baseRTT}}{\mathrm{RTT}} \mathrm{w}(t-\mathrm{RTT})+\alpha\right),
$$

where the unit time is regarded as the updating period, $w(t)$ is the congestion window, baseRTT is the minimum RTT observed, and $\alpha>0$ is the congestion control parameter. Respectively substituting $T_{i}$ and $d_{i}$ for RTT and baseRTT in (3), we obtain a discrete-time model for the FAST TCP sources:

$$
w_{i}(t+1)-w_{i}(t)=\frac{1}{2}\left(-w_{i}(t)+\frac{d_{i}}{T_{i}} w_{i}\left(t-T_{i}\right)+\alpha_{i}\right),
$$

from which, by using Euler's method, we derive a continuous-time dynamic model

$$
\dot{w}_{i}(t)=\frac{1}{2}\left(-w_{i}(t)+\frac{d_{i}}{d_{i}+q_{i}(t)} w_{i}\left(t-T_{i}\right)+\alpha_{i}\right) .
$$

Next, we develop a static model to describe the queuing delay behavior at the link. For this purpose, we need to define the sending rate of each source to calculate the total capacity consumed by all the sources. Considering that the queuing delay is generated at the link in itself, we adopt the following definition of sending rate:

$$
x_{i}(t):=\frac{w_{i}\left(t-\tau_{i}^{f}\right)}{d_{i}+p(t)},
$$

which is not estimated at the source $i$ but seen at the link at time $t$. Then, measuring the queuing delay at the source $i$, the sending rate at time $t$ can be estimated at the source $i$ as

$$
x_{i}\left(t-\tau_{b}^{i}\right)=\frac{w_{i}\left(t-\tau_{i}^{f}-\tau_{i}^{b}\right)}{d_{i}+p\left(t-\tau_{i}^{b}\right)}=\frac{w_{i}\left(t-T_{i}\right)}{d_{i}+q_{i}(t)} .
$$

Based on the defined sending rate and the self-clocking property of TCP [2], and ignoring the fast dynamics at the link, we propose a static model for the queuing delay dynamics:

$$
\sum_{i} \frac{w_{i}\left(t-\tau_{i}^{f}\right)}{d_{i}+p(t)}\left\{\begin{array}{l}
=c \text { if } p(t)>0 \\
\leq c \text { if } p(t)=0
\end{array},\right.
$$

which means that the sum of all the source's sending rate must be less than the capacity of the link. The static model (6) can be interpreted as the queuing delay at the link is algebraically determined by the delayed congestion windows of the sources. In other words, each source's congestion window and queuing delay can be regarded as the input and output of the link system respectively, and $p(t)$ is determined by $w_{i}\left(t-\tau_{i}^{f}\right)$ for $1 \leq i \leq N$. In addition, the static model (6) adequately describes the feedback delay between the congestion window of each source and queuing delay measured at each source from the viewpoint of (5). This property is shown by sustituting $t-\tau_{i}^{b}$ into $t$ in (6):

$\sum_{j} \frac{w_{j}\left(t-\tau_{j}^{f}-\tau_{i}^{b}\right)}{d_{i}+p\left(t-\tau_{i}^{b}\right)}=\frac{w_{i}\left(t-T_{i}\right)}{d_{i}+q_{i}(t)}+\sum_{j \neq i} \frac{w_{j}\left(t-\tau_{j}^{f}-\tau_{i}^{b}\right)}{d_{i}+p\left(t-\tau_{i}^{b}\right)}$

which explicitly indicates the feedback delay $T_{i}$ between $w_{i}(t)$ and $q_{i}(t)$.

Now, we look into the case that $\sum_{i} \frac{w_{i}\left(t-\tau_{i}^{f}\right)}{d_{i}}<c$ for $t<t_{0}$, that is, $q_{i}(t)=p\left(t-\tau_{i}^{b}\right)=0$ for $t<t_{0}+\tau_{i}^{b}$. In this case, the congestion window model (4) is changed to

$$
\dot{w}_{i}(t)=\frac{1}{2}\left(-w_{i}(t)+w_{i}\left(t-T_{i}\right)+\alpha_{i}\right) \text { for } t<t_{0}+\tau_{i}^{b},
$$

which implies that $w_{i}(t)$ exponentially increases until at least $\sum_{i} \frac{w_{i}\left(t-\tau_{i}^{f}\right)}{d_{i}}>c$ and $p(t)>0$, that is, the congestion occurs. Accordingly, in the subsequent analysis, we can assume that the FAST TCP sources are under congestion, and the static model for the queuing delay is simplified as

$$
\sum_{i} \frac{w_{i}\left(t-\tau_{i}^{f}\right)}{d_{i}+p(t)}=c
$$

The whole closed-loop system under congestion consists of the congestion window adjustment rule of each source described by (4) and the queuing delay described by (7). As shown in (4), the dynamic system for the congestion window adjustment is represented by $N$ number of nonlinear delay differential equations in a distributed manner. The corresponding equilibrium points $w_{i}^{*}$ and $p^{*}$ of (4) and (7) are uniquely computed as

$$
\begin{aligned}
w_{i}^{*} & =\alpha_{i}+\frac{\alpha_{i}}{\sum_{j} \alpha_{j}} c d_{i} \text { for } 1 \leq i \leq N \\
p^{*} & =\frac{\sum_{j} \alpha_{j}}{c} .
\end{aligned}
$$

\section{BOUNDEDNESS}

In this section, we investigate the boundedness properties of $w_{i}(t)$ and $p(t)$ with respect to time $t$. We show in the following lemma that $w_{i}(t)$ is uniformly bounded both below and above.

Lemma 1: The congestion window $w_{i}(t)$ described by (4) is bounded both below and above as

$\alpha_{i}+c_{1} e^{-\frac{1}{2} t} \leq w_{i}(t) \leq \alpha_{i}+c d_{i}+c_{2} e^{-\frac{1}{2} t}$ for all $t \geq 0$,

where $c_{1}:=\left(w_{i}(0)-\alpha_{i}\right)$ and $c_{2}:=\left(w_{i}(0)-\alpha_{i}-c d_{i}\right)$.

Proof: Since $\frac{d_{i}}{d_{i}+q_{i}} w_{i}\left(t-T_{i}\right) \geq 0$ in (4), we have an inequality

$$
\dot{w}_{i}(t) \geq \frac{1}{2}\left(\alpha_{i}-w_{i}(t)\right)
$$


from which we obtain that

$$
w_{i}(t) \geq \alpha_{i}+\left(w_{i}(0)-\alpha_{i}\right) e^{-\frac{1}{2} t} \text { for all } t \geq 0
$$

by applying the comparison lemma [12].

On the other hand, using (6), we derive that

$$
\frac{w_{i}\left(t-T_{i}\right)}{d_{i}+q_{i}(t)}=\frac{w_{i}\left(t-\tau_{i}^{f}-\tau_{i}^{b}\right)}{d_{i}+p\left(t-\tau_{i}^{b}\right)} \leq \sum_{j} \frac{w_{j}\left(t-\tau_{j}^{f}-\tau_{i}^{b}\right)}{d_{j}+p\left(t-\tau_{i}^{b}\right)} \leq c,
$$

which is used to obtain from (4) that

$$
\dot{w}_{i}(t) \leq \frac{1}{2}\left(c d_{i}+\alpha_{i}-w_{i}(t)\right) .
$$

By applying the comparison lemma again, we have

$$
\begin{gathered}
w_{i}(t) \leq \alpha_{i}+c d_{i}+\left(w_{i}(0)-\alpha_{i}-c d_{i}\right) e^{-\frac{1}{2} t} \\
\text { for all } t \geq 0 .
\end{gathered}
$$

Note that according to Lemma 1 , if $w_{i}(0) \leq \alpha_{i}+c d_{i}$, $w_{i}(t) \leq \alpha_{i}+c d_{i}$ for all $t \geq 0$, but if $w_{i}(0)>\alpha_{i}+c d_{i}$, the upper bound of $w_{i}(t)$ exponentially decreases to $\alpha_{i}+c d_{i}$.

We show in the following lemma that $p(t)$ is uniformly bounded above.

Lemma 2: The queuing delay $p(t)$ described by (7) is uniformly bounded above; the RTT $T_{i}(t)=d_{i}+q_{i}(t)=$ $\tau_{i}^{f}(t)+\tau_{i}^{b}$ is uniformly bounded above.

Proof: Using the identity (7) and a definition $d_{m}:=$ $\min _{i} d_{i}$, we obtain an inequality

$$
c=\sum_{i} \frac{w_{i}\left(t-\tau_{i}^{f}\right)}{d_{i}+p(t)} \leq \sum_{i} \frac{w_{i}\left(t-\tau_{i}^{f}\right)}{d_{m}+p(t)},
$$

which is rewritten as

$$
p(t) \leq \frac{1}{c} \sum_{i} w_{i}\left(t-\tau_{i}^{f}\right)-d_{m} .
$$

Using the upper bound of $w_{i}(t)$ presented in Lemma 1, we obtain that

$$
p(t) \leq \frac{1}{c} \sum_{i}\left(\alpha_{i}+c d_{i}+c_{2} e^{-\frac{1}{2} t}\right)-d_{m} .
$$

Moreover, since $q_{i}(t):=p\left(t-\tau_{i}^{b}\right), q_{i}(t)$ is also uniformly bounded above, and $T_{i}(t)$ is uniformly bounded above.

\section{GLOBAL EXPONENTIAL STABILITY}

In order to show the global exponential stability of (4) and (7), we introduce several variables defined as

$$
\begin{aligned}
w_{i}^{d}\left(\theta_{i}\right) & =w_{i}\left(t+\tau_{i}^{b}-\tau_{M}^{b}\right) \\
W_{d}(t) & :=\sum_{i} \frac{w_{i}^{d}\left(\theta_{i}\right)}{d_{i}},
\end{aligned}
$$

where $\theta_{i}:=t+\tau_{i}^{b}-\tau_{M}^{b}, \tau_{M}^{b}:=\max _{i} \tau_{i}^{b}$, and the existence of $\tau_{M}^{b}$ is guaranteed by Lemma 2. Then, using (4), we obtain the differential equation for $w_{i}^{d}\left(\theta_{i}\right)$ :

$$
\dot{w_{i}^{d}}\left(\theta_{i}\right)=\frac{1}{2}\left(-w_{i}^{d}\left(\theta_{i}\right)+\frac{d_{i} w_{i}\left(t-\tau_{i}^{f}-\tau_{M}^{b}\right)}{d_{i}+p\left(t-\tau_{M}^{b}\right)}+\alpha_{i}\right)
$$

We also need the following lemma in proving the global exponential stability of (4) and (7).

Lemma 3: $W_{d}(t)$ defined by (14) exponentially converges to its equilibrium point

$$
W_{d}^{*}=\sum_{i} \frac{w_{i}^{*}}{d_{i}}=\sum_{i} \frac{\alpha_{i}}{d_{i}}+c .
$$

Proof: Dividing both sides of (15) by $d_{i}$ and summing up for $1 \leq i \leq N$, we obtain that

$$
\begin{aligned}
& \sum_{i} \frac{\dot{w}_{i}^{d}\left(\theta_{i}\right)}{d_{i}}= \\
& \frac{1}{2}\left(-\sum_{i} \frac{w_{i}^{d}\left(\theta_{i}\right)}{d_{i}}+\sum_{i} \frac{w_{i}\left(t-\tau_{i}^{f}-\tau_{M}^{b}\right)}{d_{i}+p\left(t-\tau_{M}^{b}\right)}+\sum_{i} \frac{\alpha_{i}}{d_{i}}\right),
\end{aligned}
$$

which is converted into the following equation by using the identity (7):

$$
\sum_{i} \frac{\dot{w}_{i}^{d}\left(\theta_{i}\right)}{d_{i}}=\frac{1}{2}\left(-\sum_{i} \frac{w_{i}^{d}\left(\theta_{i}\right)}{d_{i}}+c+\sum_{i} \frac{\alpha_{i}}{d_{i}}\right),
$$

which is converted again by using the definition of $W_{d}(t)$ into

$$
\dot{W}_{d}=\frac{1}{2}\left(-W_{d}+c+\sum_{i} \frac{\alpha_{i}}{d_{i}}\right) .
$$

It is easily shown that the solution of (16) is

$$
W_{d}(t)=c+\sum_{i} \frac{\alpha_{i}}{d_{i}}+\left(W_{d}(0)-W_{d}^{*}\right) e^{-\frac{1}{2} t},
$$

which implies that $W_{d}(t)$ exponentially converges to the equilibrium point $\left(c+\sum_{i} \frac{\alpha_{i}}{d_{i}}\right)$. On the other hand, using the equilibrium points $w_{i}^{*}$ represented in (8), we derive that

$$
\sum_{i} \frac{w_{i}^{*}}{d_{i}}=\sum_{i} \frac{\alpha_{i}+c \frac{\alpha_{i} d_{i}}{\sum_{i} \alpha_{i}}}{d_{i}}=\sum_{i} \frac{\alpha_{i}}{d_{i}}+c,
$$

which completes the proof.

Now, we establish the global exponential stability of FAST TCP (4) and (7) in the following theorem.

Theorem 1: FAST TCP described by (4) and (7) is globally exponentially stable provided that

$$
\alpha_{i}>0 \text { for } 1 \leq i \leq N .
$$

Proof: First, we prove by showing a contradiction that $w_{i}(t)$ of each source converges to a constant value. Then, we show that $w_{i}(t)$ of each source exponentially converges to its equilibrium point by using Lemma 3 .

Suppose that some of $w_{i}(t)$ for $1 \leq i \leq N$ do not converge to certain constant values. Then, the boundedness of $w_{i}(t)$ from Lemma 1 implies that each $w_{i}(t)$ that does not converge should oscillate with respect to time $t$. Moreover, the convergence of $W_{d}(t)$ from Lemma 3 implies that at least two of $w_{i}(t)$ for $1 \leq i \leq N$ should oscillate with the identical period, and the phase difference between the two oscillating $w_{i}(t)$ 's should be half the period.

Closely looking into (4), it is easily shown that the period should be $T_{i}$ for the source $i$ to oscillate. However, every 


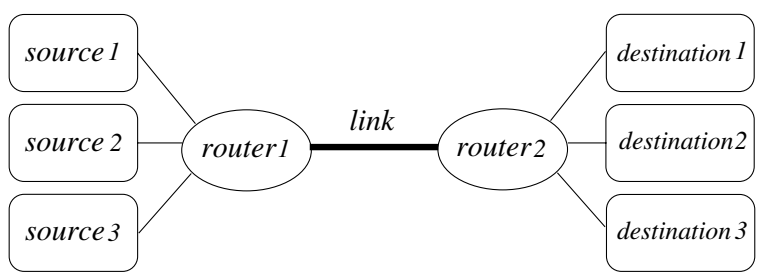

Fig. 1. Network topology

$T_{i}:=d_{i}+q_{i}(t)$ for $1 \leq i \leq N$ is different from each other as long as every $d_{i}$ is different from each other, which shows a contradiction. Therefore, we prove that $w_{i}(t)$ of each source converges to a constant value.

On the other hand, since the equilibrium points $w_{i}^{*}$ for $1 \leq i \leq N$ represented in (8) are unique and $W_{d}(t)$ in Lemma 3 exponentially converges, we can conclude that $w_{i}(t)$ exponentially converges to its equilibrium point $w_{i}^{*}=$ $\alpha_{i}+c \frac{\alpha_{i} d_{i}}{\sum_{j} \alpha_{j}}$.

Corollary 1: The queuing delay $p(t)$ described by (7) exponentially converges to its equilibrium point $p^{*}=\frac{\sum_{j} \alpha_{j}}{c}$.

Proof: Using (8) and (9), we get an identity

$$
\sum_{i} \frac{w_{i}^{*}}{d_{i}+p^{*}}=c
$$

and substituting this identity for $c$ in (7), we derive that

$$
\sum_{i}\left(\frac{w_{i}\left(t-\tau_{i}^{f}\right)}{d_{i}+p(t)}-\frac{w_{i}^{*}}{d_{i}+q^{*}}\right)=0 .
$$

The congestion window of each source $w_{i}\left(t-\tau_{i}^{f}\right)$ in (18) exponentially converges to its equilibrium point as shown in Theorem 1; simultaneously, the identity (18) should hold whenever $\sum_{i} \frac{w_{i}\left(t-\tau_{i}^{f}\right)}{d_{i}}>c$. Consequently, $p(t)$ in (18) should also exponentially converge to its equilibrium point $p^{*}=$ $\frac{\sum_{j} \alpha_{j}}{c}$.

Theorem 1 shows that the closed-loop system (4) and (7) is globally exponentially stable as long as $\alpha_{i}>0$ for $1 \leq i \leq$ $N$. It is remarkable that the global exponential stability of FAST TCP is achieved without any specific condition even in the presence of the network feedback delay.

\section{Simulation AND EXPERIMENT}

We simulate FAST TCP described by (4) and (7) with not only MATLAB but also $n s-2$ network simulator [13]. The $n s$-2 implementation of FAST TCP is found in [11].

We conduct the simulations for a network with a single bottleneck link shared by three heterogeneous sources as shown in Fig. 1. The link capacity and the round trip latencies are set as $c=150 \mathrm{pkts} / \mathrm{ms}$ and $d_{1}=50 \mathrm{~ms}, d_{2}=70 \mathrm{~ms}$, $d_{3}=90 \mathrm{~ms}$. The congestion control parameters are set as $\alpha_{1}=200, \alpha_{2}=250, \alpha_{3}=300$, which are small values in comparison with the delay-bandwidth product of each source.

Table I summarizes the calculated equilibrium points of each source adopted in the simulation, and Fig. 2 shows the
TABLE I

EQUILIBRIUM POINTS

\begin{tabular}{|c|c|c||c|c|}
\hline $\begin{array}{c}c \\
(\mathrm{pkts} / \mathrm{ms})\end{array}$ & $\begin{array}{c}d \\
(\mathrm{~ms})\end{array}$ & $\begin{array}{c}\alpha \\
(\mathrm{pkts})\end{array}$ & $\begin{array}{c}\text { Window size } \\
(\mathrm{pkts})\end{array}$ & $\begin{array}{c}\text { Queuing delay } \\
(\mathrm{ms})\end{array}$ \\
\hline \hline \multirow{3}{*}{150} & 50 & 200 & 2200 & \multirow{2}{*}{5} \\
\cline { 2 - 4 } & 70 & 250 & 3750 & \multirow{2}{*}{5} \\
\cline { 2 - 4 } & 90 & 300 & 5700 & \\
\hline
\end{tabular}

simulation results of MATLAB and $n s-2$ simulator, where the broken lines indicate the MATLAB results and the solid lines indicate the $n s-2$ results. All figures illustrate that each source's congestion window is uniformly bounded and globally exponentially stable provided that $\alpha_{i}>0$. Moreover, the similarities of the shapes between MATLAB and $n s-2$ results in Fig. 2 illustrate that the proposed model (4) and (7) for the FAST TCP sources and the queuing delay adequately describes the real network consisting of the FAST TCP sources.

\section{CONCLUSIONS}

In this paper, we analyze the global exponential stability of FAST TCP in a single-link multi-source network in the presence of the network feedback delay. We propose a continuous-time model for the FAST TCP sources and a static model for the link. The proposed model turns out to satisfy the self-clocking property of TCP and indicate the network feedback delay explicitly. Based on this network model, we show that FAST TCP is globally exponentially stable provided that each source's control parameter $\alpha_{i}$ is positive. It is remarkable that FAST TCP is globally exponentially stable under the trivial condition even in consideration of the network feedback delay. The simulation results illustrate the validity of the proposed model and the global exponential stability of FAST TCP.

\section{REFERENCES}

[1] Raj Jain, K. K. Ramakrishnan and Dah-Ming Chiu, "Congestion Avoidance in Computer Networks with a Connectionless Network Layer," Technical Report DEC-TR-506, Digital Equipment Corporation, August 1987.

[2] V. Jacobson, "Congestion Avoidance and Control," In Proceedings of ACM SIGCOMM'88, August 1988.

[3] C. V. Hollot, V. Misra, D. Towsley, and W. B. Gong, "Analysis and Design of Controllers for AQM Routers Supporting TCP Flows," IEEE Transactions on Automatic Control, Vol. 47, No. 6, pp. 945-959, 2002.

[4] Steven H. Low, Fernando Paganini, Jiantao Wang and John C. Doyle, "Linear Stability of TCP/RED and a Scalable Control," Computer Networks Journal, Vol. 43, No. 5, pp. 633-647, December 2003.

[5] Cheng Jin, David X. Wei and Steven H. Low, "FAST TCP: motivation, architecture, algorithms, performance," In Proceedings of IEEE Infocom, March 2004. http: / / netlab.caltech.edu/FAST/

[6] Sally Floyd, "HighSpeed TCP for Large Congestion Windows," RFC 3649, Experimental, December 2003.

http: / /www.icir.org/floyd/hstcp.html

[7] Tom Kelly, "Scalable TCP: Improving Performance in High-speed Wide Area Networks," ACM SIGCOMM Computer Communication Review, Vol. 33, No. 2, pp. 83-91, April 2003.

[8] Lisong $\mathrm{Xu}$, Khaled Harfoush and Injong Rhee, "Binary Increase Congestion Control for Fast Long-distance Networks," In Proceedings of IEEE Infocom, March 2004.

[9] Jiantao Wang, David X. Wei and Steven H. Low, "Modelling and Stability of FAST TCP," In Proceedings of IEEE Infocom, April 2005. 


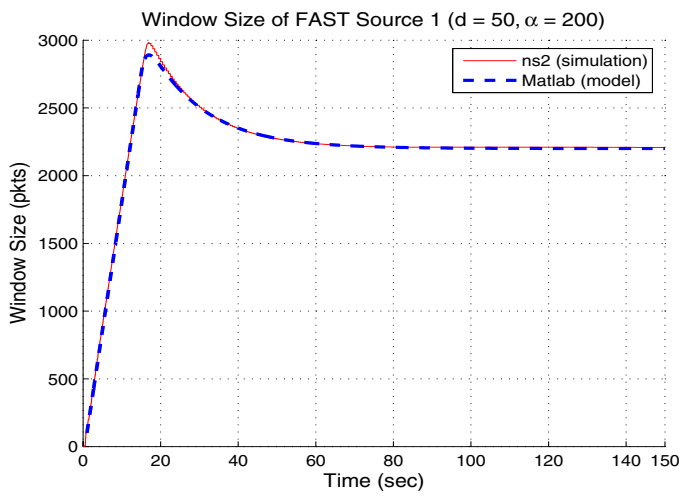

(a) Window size of FAST TCP source 1

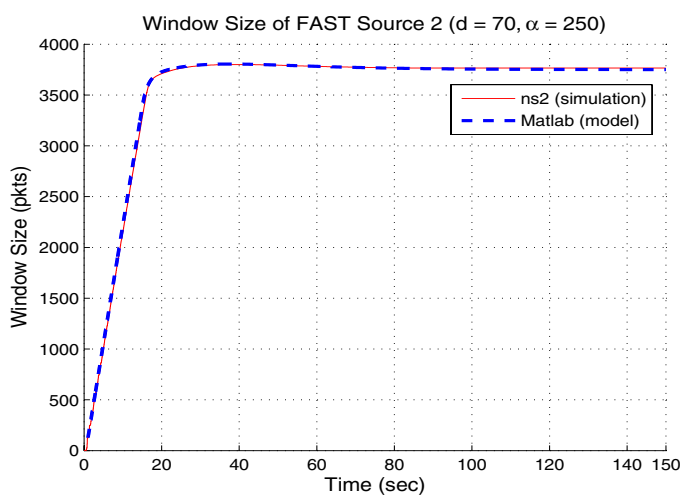

(b) Window size of FAST TCP source 2

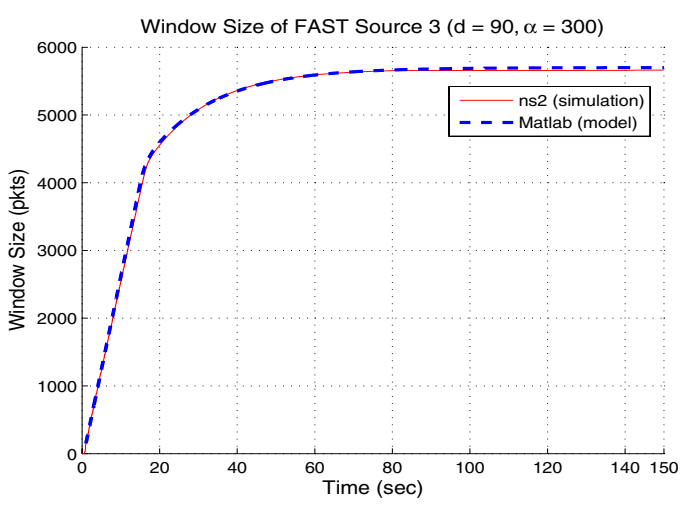

(c) Window size of FAST TCP source 3

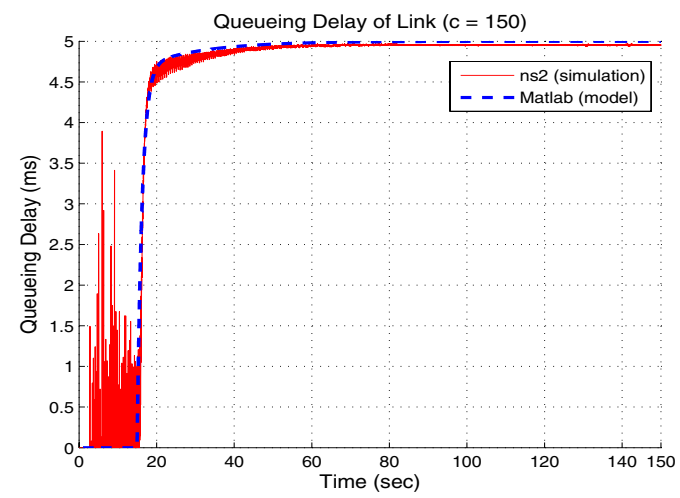

(d) Queuing delay of link

Fig. 2. MATLAB and $n s-2$ simulation results of the FAST TCP network
[10] Joon-Young Choi, Kyungmo Koo, Jin S. Lee and Steven H. Low, "Global Stability of FAST TCP in Single-Link Single-Source Network," In Proc. 44th IEEE Conf. Decision and Control, Seville, Spain, Dec. 2005.

[11] FAST TCP Simulator Module for ns-2 version 1.1. http: / / www. cubinlab.ee.mu.oz.au/ns2 fasttcp/

[12] Hassan K. Khalil, Nonlinear Systems, 3rd ed., Prentice Hall, 2002.

[13] The Network Simulator - ns-2. http: //www.isi.edu/nsnam/ns/ 